\title{
Simulations of composite carbon films with nanotube inclusions
}

\author{
M. G. Fyta and P. C. Kelires \\ Physics Department, University of Crete, P.O. Box 2208, 710 03, Heraclion, Crete, Greece
}

(Dated: November 11, 2018)

\begin{abstract}
We study the interfacial structure, stability, and elastic properties of composite carbon films containing nanotubes. Our Monte Carlo simulations show that Van der Waals forces play a vital role in shaping up the interfacial geometry, producing a curved graphitic wall surrounding the tubes. The most stable structures are predicted to have intermediate densities, high anisotropies, and increased elastic moduli compared to pure amorphous carbon films.
\end{abstract}

Interest on nanocomposite carbon systems is steadily growing for both practical and fundamental reasons. These materials combine the properties of carbon nanostructures with those of the embedding medium, which usually is an amorphous carbon (a-C) matrix. By appropriately choosing the type, size, and positioning of the nanostructures, one aims at controlling the mechanical and optoelectronic properties of the composite system.

From a fundamental point of view, it is vital to understand how the nanostructures interact with the embedding matrix, to unravel the structural elements at the interface, and to examine their influence on the properties of the material. A very interesting case arises when the composite contains nanostructures which, in the absence of a matrix, are assembled by long-range Van der Waals (VdW) forces. For example, films containing nanotube fragments and fullerene-like inclusions have been produced, and reported to have high hardness and high elastic recovery. 1] One of the unknown factors in this case is how the matrix, a purely covalent material, bonds to the nanostructure. This issue, although important for the optimization of nanocomposite films, has not been addressed so far at the atomistic level.

We report here the first direct simulations of a prototypical nanocomposite material, made up of carbon nanotubes (CNT) inside an a-C matrix. Our goal is to clearly identify the interfacial structure, and to infer the stability and hardness of the material. A computational approach to this problem needs to take into account not only shortrange covalent forces, which are sufficient for the description of the interactions within either the CNT's and the a-C matrix, but also the weaker long-range VdW forces, which might be important for the interactions between the CNT's and the matrix. In order to capture the subtle effects of such forces on the structure of the system, we need to consider simulational cells of realistic dimensions (thousands of atoms.) This excludes the use of either $a b$ initio or tight-binding schemes.

We therefore utilize a less sophisticated computational scheme based on empirical potentials, which is however capable of describing large systems and correctly introducing VdW forces. For the intra-tubule and intra-matrix short-range interactions, we use the Tersoff potential, 2] which provides a fairly good description of
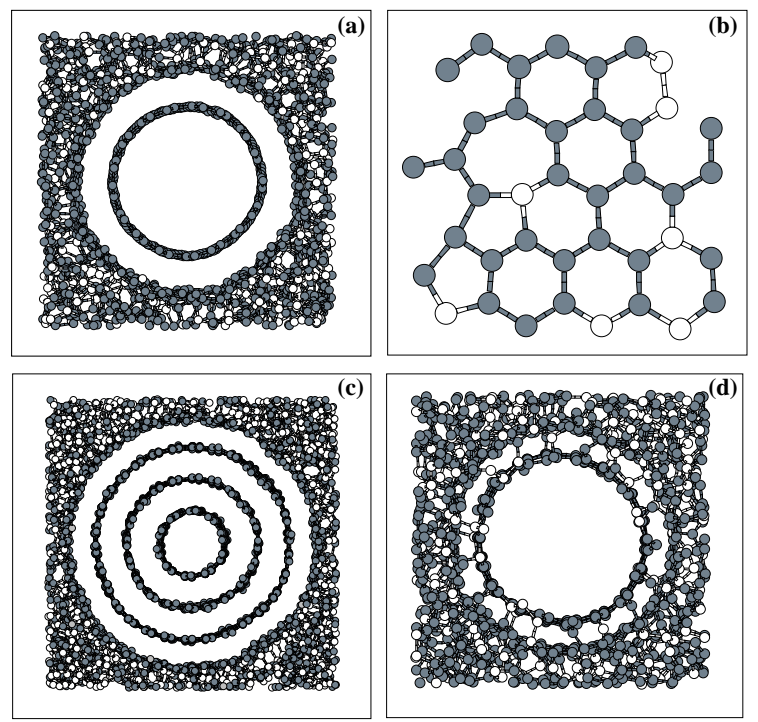

FIG. 1: Cross sections from ball and stick models of carbon nanotube composite structures. (a) An embedded SWCN. (b) Part of the curved graphitic wall surrounding the SWCN. (c) An embedded MWCN. (d) A structure formed without VdW interactions. Filled (open) spheres denote $\mathrm{sp}^{2}\left(\mathrm{sp}^{3}\right)$ atoms.

the structure and energetics of a wide range of carbon materials. [3, 4, 5] We confirmed that the potential reproduces the high elastic moduli of single-wall carbon nanotubes (SWCN's). For example, individual SWCN's with diameters of $\sim 1 \mathrm{~nm}$ exhibit bulk moduli of $\sim 200$ $\mathrm{GPa}$, in very good agreement with ab initio results. $[$ ]

For the interactions between the CNT's and the matrix, we use a simple Lennard-Jones potential, [] which was shown to succesfully describe the bulk properties of solid $C_{60}[7]$ and multiple-shell carbon fullerenes. [8] We limit the attractive interactions between atoms of the nanotube and atoms in the matrix within a cutoff distance of $0.8 \mathrm{~nm}$. Interactions beyond this distance contribute negligibly to the energy.

The composite structures are generated using a continuous-space Monte Carlo (MC) approach, extensively described in the past. [4, 5] The initial structure to start with is a cubic diamond crystal with an empty cylindrical core of a predetermined diameter, formed by artificially removing the atoms within this volume, into 
which the chosen CNT is inserted. The amorphous matrix is then generated by melting and subsequent quenching of the surrounding diamond atoms, while keeping the atoms of the CNT frozen in their ideal positions. Finally, full relaxation of the whole structure takes place, both in the atomic positions and the volume (density) of the cell. Properties are calculated by $\mathrm{MC}$ ensemble averaging at $300 \mathrm{~K}$.

Matrices of various densities (mean coordination) are formed by appropriately choosing the size of the initial cell. The CNT's have open ends and extend through the entire length of the cube. Because periodic boundary conditions (PBC) are applied to the cells, this corresponds to CNT's of infinite length. The size of the cells ranges from about 1.5 to $4.0 \mathrm{~nm}$. Nanotubes of various chiralities were embedded, with diameters ranging from about 0.8 to $2.8 \mathrm{~nm}$. Due to the PBC, this corresponds to a dense array of CNT's packed in parallel. Although this probably is an idealized model of a CNT nanocomposite, it provides the essential features of the CNT-matrix interaction. The system resembles CNT bundles, but with a-C material in between the tubes.

Representative nanocomposite structures formed this way are portrayed in Fig. 1. Panel (a) shows a cross section of a $(7 \times 10)$ SWCN, having a diameter of 1.2 $\mathrm{nm}$, embedded in a matrix with mean coordination $\bar{z}=$ $3.24\left(\right.$ density $\left.=2.29 \mathrm{gcm}^{-3}\right)$. The remarkable feature in the structure is that the atoms of the surrounding matrix reconstruct in such a way as to form an outer wall concentric to the nanotube. A double-wall nanotube is effectively formed. The outer wall is a curved graphitic sheet, but with some degree of disorder, as a close inspection of its structure reveals. Part of this sheet is depicted in panel (b). A ring statistics analysis shows that the sheet mainly consists of six-fold rings, their fraction being considerably higher than the corresponding fraction in the rest of the matrix. However, odd-membered rings do exist, necessitated by the presence of some $\mathrm{sp}^{3}$ atoms in the wall, which serve as bridges with the rest of the matrix.

In the lowest-energy structures (see below), the optimum distance between the outer wall and the CNT is close to the graphite interplanar one $(0.34 \mathrm{~nm})$. In less stable structures, it deviates from this value depending on the matrix density. This distance is independent of the tube diameter. A similar pattern is also observed in structures with embedded multi-wall carbon nanotubes (MWCN's), as shown in Fig. 1(c). The interaction of the MWCN with the matrix forms again a graphitic sheet. The outer interplanar distance is the same with the inner interwall separations in the MWCN. The phenomenon is independent of the number of sheets in the MWCN.

Note that in all cases the interaction of the matrix graphitic shell with the CNT's does not involve any covalent bonding. In order to unravel the contributions of short- and long-range forces to the formation of this wall,

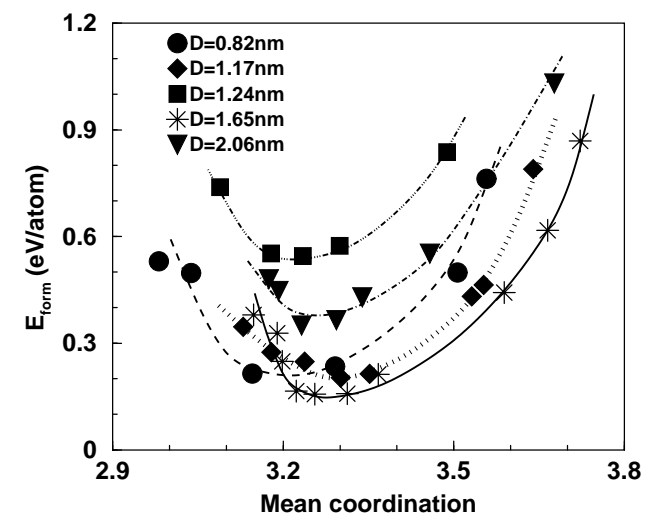

FIG. 2: Formation energy of SWCN's of various diameters versus mean coordination of the embedding matrix. Lines are fits to the points.

we generated structures by only using the short-ranged Tersoff potential for the interactions through out the system, thus "turning off" the VdW interactions. One of these structures is shown in Fig. 1(d). Again, an outer wall is being formed, but it is defective. Many covalent bridge bonds are generated between the matrix wall and the SWCN, and so the interwall distance necessarily approaches the value of $\sim 0.15 \mathrm{~nm}$, much smaller than the interplanar distance in graphite or in MWCN's, and close to the covalent bond length value.

This indicates that the repulsive forces of the potential tend to drive the matrix and CNT atoms apart, but its attractive forces impose these artificial bridge bonds, which necessitate the transformation of ideal $\mathrm{sp}^{2}$ sites on the nanotube surface into distorted $\mathrm{sp}^{3}$ sites, as shown in the plot. We conclude that short-range attractive forces are not involved in the matrix-CNT interaction, and that the VdW forces, although weak, $[9]$ are driving the perfect reconstruction of the matrix atoms while keeping intact the ideal CNT geometry.

Stability is a crucial property of any composite system. As a measure of the stability of CNT's in a-C, we calculated their formation energy, 5 ] defined as the energy of the whole structure compared to the sum of the energies of its constituents (the a-C matrix and the nanostructure.) The formation energies $\mathrm{E}_{\text {form }}$ of a number of SWCN's of various diameters versus the matrix coordination are plotted in Fig. 2. Well defined minima exist for matrices of $\bar{z} \simeq 3.2-3.3$, corresponding to densities of about $2.3-2.4 \mathrm{~g} \mathrm{~cm}^{-3}$. The denser (or diluted) the matrix the less stable the system becomes. Indeed, annealing at elevated temperatures results in heavy distortion of CNT's when embedded in dense (diluted) matrices, while nanotubes in matrices with $\bar{z}$ near the minima retain their optimal shape.

For dense matrices, this result is understood by noting that the denser the a-C matrix the fewer the $\mathrm{sp}^{2}$ sites, 


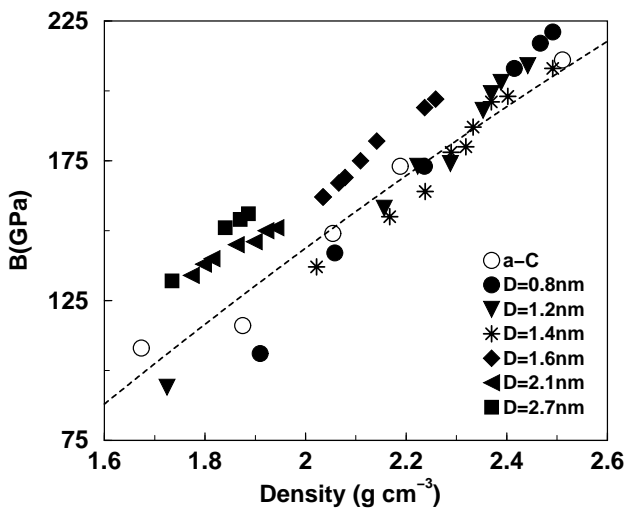

FIG. 3: Bulk moduli of SWCN's of various diameters versus nanocomposite density. Dashed line is a fit to a-C values.

and the formation of a graphitic wall to interact via $\mathrm{VdW}$ forces with the CNT becomes less easy. In fact, the wall in such cases is higly defective, with many $\mathrm{sp}^{3}$ sites, and its distance to the CNT shrinks to $\sim 0.24-0.28 \mathrm{~nm}$. The incomplete wall - CNT interaction results in CNT distortions. Both deformations in the wall and the CNT increase the energy. Diluted a-C matrices, on the other hand, are characterized by a sizeable fraction of $\mathrm{sp}^{1}$ sites, which act as defects on a graphitic plane. We find an increasing number of $\mathrm{sp}^{1}$ sites on the wall with decreasing density. This again destroys locally the curved graphitic nature, increasing the energy.

Finally, we have examined the elastic properties of this nanocomposite system. A representative quantity is the bulk modulus $B$. We calculated $B$ for several structures containing CNT's of various diameters. Fig. 3 shows the calculated moduli plotted as a function of the nanocomposite density. As a comparison, calculated moduli of single-phase a-C films are also shown. We observe a systematic increase of the nanocomposite $B$, with respect to a-C, when it contains tubes of larger diameter, while smaller tubes induce increase only when embedded in dense matrices.

This behavior is the result of a compromise between two competing factors, i.e., the matrix density and the tube diameter. Because we have a high density of tubes in the medium, their size becomes a crucial factor. The larger the tube diameter the smaller the contribution of the matrix to the material's $B$, and vice versa. By having larger tubes at low densities, the contribution of the soft matrix declines, and that of the tubes strengthens the material. Smaller embedded tubes operate in the opposite direction, despite the fact that the moduli of isolated CNT's increase as their diameter decreases. In more dense and rigid matrices, inclusion of smaller tubes enhances $B$ both because such tubes are very rigid and because the matrix contribution dominates. Also, our calculations show that $B$ is independent of CNT chiralities.
Another marked characteristic of these nanocomposites is their high elastic anisotropy. We show this by calculating the components of the Young's modulus in the direction of the tube (axial) $Y_{\text {axial }}$ and in the transverse directions $Y_{\text {trans. }}$. We find that in all cases the anisotropy, defined by the ratio $A=Y_{\text {trans }} / Y_{\text {axial }}$, approaches that for the isolated SWCN. For example, a SWCN with a diameter of $0.8 \mathrm{~nm}$ has $Y_{\text {axial }}=1100$ GPa and $Y_{\text {trans }}=620 \mathrm{GPa}$, yielding $A=0.56$, while the nanocomposite with this tube and a matrix of density $2.6 \mathrm{gcm}^{-3}$ has $Y_{\text {axial }}=570 \mathrm{GPa}, Y_{\text {trans }}=380 \mathrm{GPa}$, and $A=0.67$. The anisotropy increases with increasing tube diameter (tube volume fraction), because the tube contribution overwhelms the isotropic matrix part. Another measure of elastic anisotropy is provided by the factor $\eta=2 C_{44} /\left(C_{11}-C_{12}\right)$. A value of unity corresponds to a completely isotropic medium. By calculating the elastic constants for a number of nanocomposites, we confirmed their high anisotropy. For example, the structure shown in Fig. 1(a) has $\eta=0.75$. All pure a-C networks have $\eta \simeq$ 0.98. A more detailed account of the interesting elastic properties of embedded tubes will be given elsewhere.

The interplay between the nanotube size and the embedding density gives one the opprortunity to tailor the mechanical properties of this nanocomposite system. It is striking that higher moduli are achieved by having the tubes in a "bundle" arrangement without the need to interlink them. [1] In addition, the CNT composite material exhibits much higher bulk moduli than nanotube bundles $(\sim 40 \mathrm{GPa}),[\underline{6}]$ and is expected to also have high elastic recovery. These superior mechanical properties make it suitable for many practical applications.

This work is supported by a grant from the EU and the Ministry of National Education and Religious Affairs of Greece through the action "EПEAEK" (programme

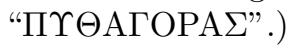

[1] G. A. J. Amaratunga, M. Chhowalla, C. J. Kiely, I. Alexandrou, R. Aharonov, and R. M. Devenish, Nature 383, 321 (1996).

[2] J. Tersoff, Phys. Rev. Lett. 61, 2879 (1988).

[3] D. Vanderbilt and J. Tersoff, Phys. Rev. Lett. 68, 511 (1992).

[4] P. C. Kelires, Phys. Rev. Lett. 73, 2460 (1994); Phys. Rev. B 62, 15686 (2000).

[5] M. G. Fyta, I. N. Remediakis and P. C. Kelires, Phys. Rev. B 67, 035423 (2003).

[6] S. Reich, C. Thomsen, and P. Ordejón, Phys. Rev. B 65, 153407 (2002).

[7] J. P. Lu, X. -P. Li and R. M. Martin, Phys. Rev. Lett. 68, 1551 (1992).

[8] J. P. Lu and W. Yang, Phys. Rev. B 49, 11421 (1994).

[9] We estimate the contribution of VdW forces to the cohesive energy of the system to be $\sim 0.2 \%$. 